\title{
Adaptive neural networks for model updating of structures
}

\section{C Chang, T Y P Chang and Y G Xu}

Department of Civil Engineering, Hong Kong University of Science and Technology, Clear Water Bay, Kowloon, Hong Kong, People's Republic of China

Received 10 June 1999, in final form 14 September 1999

\begin{abstract}
A model updating methodology based on an adaptive neural network (NN) model is proposed in this study. The NN model has a feedforward architecture and is first trained off-line using some training data that are obtained from finite-element analyses and contain modal parameters as inputs and structural parameters as outputs. To reduce the number of training data while maintaining the data completeness, the variation of structural parameters is arranged using an orthogonal array. This NN model is then adaptively retrained on-line during the model updating process in order to eliminate the difference between the measured and the predicted modal parameters. A modified back-propagation algorithm is developed, in which the learning rate is dynamically adjusted once every few iterations. A jump factor is introduced to overcome the numerical difficulty caused by the saturation of the sigmoid function in order to improve the convergence performance of the NN model. The current adaptive $\mathrm{NN}$ updating procedure is applied to a suspension bridge model and verified both numerically and experimentally. The results indicate that by adaptively training the NN model and iteratively adjusting the structural parameters, it is possible to reduce the differences between the measured and the predicted frequencies from a maximum of $17 \%$ to $7 \%$ for the first eight vertical modes.
\end{abstract}

\section{Introduction}

A precise prediction of dynamic response of a structure by finite-element (FE) analysis is necessary at least in two application areas: (a) for the employment of viable structural health assessment methodologies and (b) for the development of useful structural control strategies. In both applications, it is imperative to correctly understand the dynamic behaviour of a structure and such behaviour depends largely on the modal properties that include natural frequencies, mode shapes and damping ratios.

Many model updating methods have been developed during the last two decades. They can be roughly classified into the direct methods and the sensitivity-based methods [1]. Some typical direct methods include the optimal matrix updating methods [2], the matrix mixing methods [3], the eigen-structure assignment methods [4] and the inverse eigenvalue methods [5]. These methods directly update the total mass and/or stiffness matrices obtained from the finiteelement modelling of a structure based on some measured modal parameters. The updated mass and stiffness matrices are generally fully populated and the entries of these matrices are difficult to interpret physically. The sensitivity-based methods, on the other hand, use sensitivity matrices that correlate the perturbation between the modal parameters and the structural parameters to be updated during the updating process. Some specific methods developed along this line include the iterative methods [6], the optimization methods [7] and the Bayesian estimation methods [8], etc. These methods can preserve the structural connectivity in the updated model and provide a direct physical explanation for the updated results. When physical parameters of a structure are selected as the updated parameters, the updated results from the sensitivity-based methods can be used as a reference for damage identification [9]. Some frequent problems encountered using these methods are that the calculation of the sensitivity matrices is usually computationally intensive [10] and the matrices are often ill-conditioned [11]. Some special algorithms have been developed to improve the updating results, for example, the regularization methods [12] and the QR-orthogonal decomposition methods [13]. Despite these developments, the updating process is still computationally complex and time consuming.

With the development of artificial intelligent techniques, the neural network $(\mathrm{NN})$ methods have recently become widely accepted in the civil engineering area. Adeli and Yeh [14] presented a NN model of machine learning in engineering design. Wu et al [15] have demonstrated the application of a NN model in the detection of structural damage. The applications of NN models in structural engineering have also been done by researchers such as Hajela and Berke [16], Masri et al [17], Stephen and Vanluchene [18], Elkordy et al [19], etc. As to the application in model updating, Atalla and Inman [20] used frequency domain data to train a $\mathrm{NN}$ model; the trained $\mathrm{NN}$ model can estimate the updated parameters quickly and 
yield a model representative of the measured data. They verified the proposed method on a frame structure with noisy, experimental data, and reported that the particular choice of input data can make the training more robust with respect to noise. Levin and Lieven [21] found that the NN model is robust and can withstand the presence of noise in experimental data, and that the $\mathrm{NN}$ approach can avoid the common problem of coordinate incompleteness. The inputs and outputs of a NN model can be selected with a certain flexibility, which provides the possibility of the direct updating of the structural parameters and boundary conditions by supplying only a limited number of modal parameters from the measurement. Instead of dealing with the sensitivity matrix that might be potentially illconditioned, the NN model requires proper training using simulated or measured data. The training process could be very time-consuming and the accuracy of the predictions of this trained NN model depend very much on the training data. However, once the model is properly trained, the NN calculation is relatively fast regardless of the complexity of the structure to be updated. In addition, the NN technique is well known for its ability to model nonlinear and complex relationship that is exactly the case between the structural parameters and the modal properties.

The application of the NN methods in the area of model updating and damage identification appears to be still at its early stage. Most of the numerical examples presented up till now are confined to simple structures such as cantilever beams [21] and frames [20]. As the complexity of the structure and the number of structural parameters to be updated increase, problems associated with the NN model will arise accordingly and this issue needs to be addressed. Also, as the number of structural parameters to be updated increases, the number of training samples required to ensure sample completeness increases exponentially. A large number of training samples would inevitably require a longer training time and a more efficient training algorithm.

In our present study, a model updating methodology based on an adaptive NN model is proposed. The NN model has a feedforward architecture and is first trained off-line using some training data that are obtained from FE analyses and contain modal parameters as inputs and structural parameters as outputs. To reduce the number of training data while maintaining the data completeness, the variation of structural parameters is arranged using an orthogonal array. This NN model is then adaptively retrained on-line during the model updating process in order to eliminate the difference between the measured and the predicted modal parameters. A modified back-propagation (BP) algorithm is developed, in which the learning rate is dynamically adjusted once every few iterations, and a jump factor is introduced to overcome the numerical difficulty caused by the saturation of the sigmoid function in order to improve the convergence performance of the NN model. The current adaptive NN updating procedure is applied to a suspension bridge model and verified both numerically and experimentally.

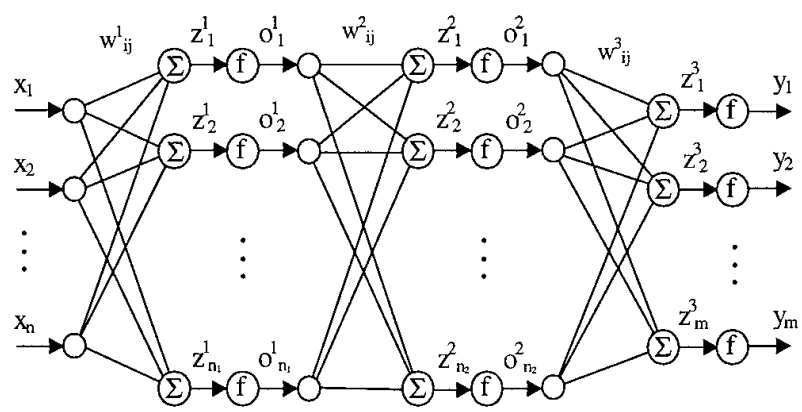

Input layer $\quad 1^{\text {st }}$ hidden layer $\quad 2^{\text {nd }}$ hidden layer $\quad$ Output layer

Figure 1. A NN model with two hidden layers.

\section{Adaptive NNs}

\subsection{Network architecture and updating strategy}

$\mathrm{NN}$ refers to one type of computational model that consists of hidden-layer neurons connected between input and output neurons. The connections between neurons are described by weights, which are to be determined through training. Linear or nonlinear functions can be assigned to the neurons to enhance the modelling flexibility. The NN model can be used to model complex nonlinear relationships, such as model updating, where explicit formulae are difficult to obtain. Figure 1 shows a feedforward NN model with $n$ inputs $(X=$ $\left.\left\{x_{i}, i=1, \ldots, n\right\}\right), m$ outputs $\left(\boldsymbol{Y}=\left\{y_{i}, i=1, \ldots, m\right\}\right)$ and two hidden layers. The numbers of inputs and outputs used in the NN model depend implicitly on the type of problem under study. Masri et al [17] showed that two hidden layers could be sufficient in most of the structure-related problems. The numbers of neurons in the hidden layers are determined normally by numerical experimentation (trial and error). The inputs considered in this study include modal parameters such as the natural frequencies $f_{i}$ 's and the mode shapes $\phi_{i}$ 's. The outputs on the other hand are the structural parameters to be updated such as modulus of elasticity $E$, mass density $\rho$ and cross sectional area $A$, etc. The objective of the NN model is to predict the structural parameters by inputting some measured modal parameters. Mathematically, the NN model represents a nonlinear mapping between inputs and outputs via the following equation,

$$
\boldsymbol{Y}=g(\boldsymbol{W}, \boldsymbol{X}) .
$$

The weight matrix $\boldsymbol{W}$ consists of weight coefficients $w_{i j}^{k}$,

$$
\boldsymbol{W}=\left\{w_{i j}^{k}, i=1, \ldots, n_{i} ; j=1, \ldots, n_{j} ; k=1,2,3\right\}
$$

where $n_{i}$ and $n_{j}$ are the numbers of neurons for the $i$ th and the $j$ th layers, respectively. The $\mathrm{NN}$ updating strategy proposed in this study include (i) the initial training of the NN model using some training data and (ii) the updating of structural parameters by the trained $\mathrm{NN}$ model simultaneously with the adaptive re-training of the $\mathrm{NN}$ model.

The purpose of the initial training is to establish a preliminary nonlinear mapping relationship between the modal parameters and the structural properties (see figure 2). 


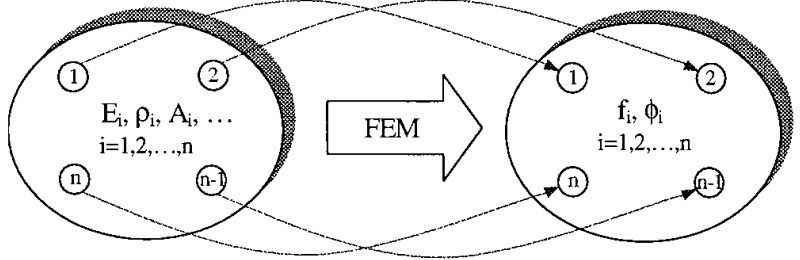

Structural Parameters

Modal Parameters

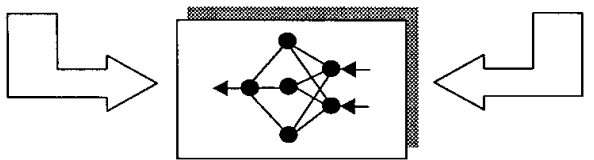

Training of the NN model

Figure 2. Initial training of the $\mathrm{NN}$ model.

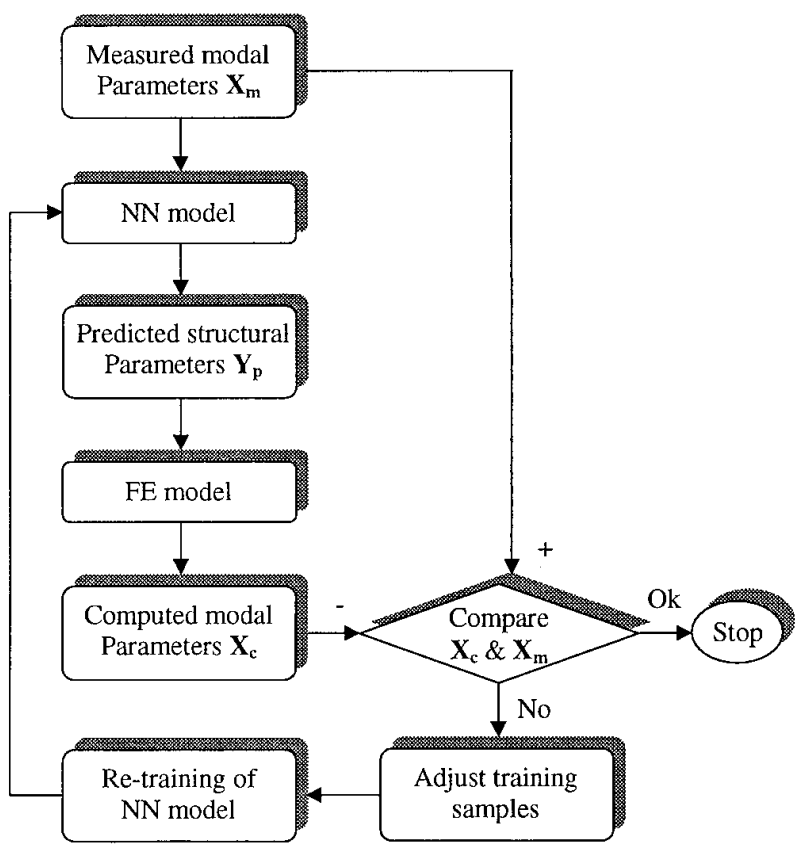

Figure 3. Structural parameters updating with the re-training of the NN model.

The training samples consist of a few sets of assumed structural parameters and their corresponding modal parameters obtained from the FE analyses. Details on the selection of training samples will be discussed in section 2.3.

After the initial training of the NN model, the model updating procedure begins with feeding measured modal parameters $\boldsymbol{X}_{m}$ into the NN model (see figure 3). The outputs of the NN model are the predicted structural parameters $\boldsymbol{Y}_{p}$. These predicted structural properties are then fed into the FE model to produce a set of calculated modal parameters $\boldsymbol{X}_{c}$. A comparison between the calculated modal parameters $\boldsymbol{X}_{c}$ and the measured modal parameters $\boldsymbol{X}_{m}$ is made based on a given criterion. If these two sets of parameters differ significantly such that the criterion is not satisfied, then the NN model will be retrained on-line using training samples that contain $\boldsymbol{X}_{c}$ and $\boldsymbol{Y}_{p}$. The re-trained NN model is then used to predict structural parameters by feeding in the measured modal parameters $\boldsymbol{X}_{m}$. The model updating and on-line retraining procedure is repeated until the difference between the calculated and the measured modal parameters satisfies the given criterion.

The basic strategy for this NN-based model updating procedure is simple and straightforward. There are however two key factors governing the success and the speed of convergence using this approach. The first is that the NN model should have a proper network architecture and a good learning algorithm to ensure that the model will converge in a speedy fashion. Second is that the training samples, including both the initial training samples and the retraining samples, should be carefully selected to reflect the inverse dynamic characteristics of the structure.

\subsection{Training algorithms}

Most of the training algorithms for the multilayer perceptron $\mathrm{NN}$ models are based on the BP method. The convergent speed of this method is usually slow. In this paper, a modified BP training algorithm with a dynamically adjusted learning rate and a jump factor is adopted.

The training of a NN model is the process of determining the weight matrix $\boldsymbol{W}$ such that the error norm $E_{r}$ between the predicted output vector $Y_{p}$ and the targeted output vector $\boldsymbol{Y}_{t}$, defined as

$$
E_{r}(\boldsymbol{W})=\left\|\boldsymbol{Y}_{p}-\boldsymbol{Y}_{t}\right\|_{2}
$$

becomes smaller than a tolerable value. The operator $\|\cdot\|_{2}$ represents the vector norm. The weight matrix is adjusted iteratively based on the following equations [22].

$$
\begin{gathered}
\boldsymbol{W}^{r+1}=\boldsymbol{W}^{r}+\Delta \boldsymbol{W}^{r} \\
\Delta \boldsymbol{W}^{r}=-\left.\eta \frac{\partial E_{r}}{\partial \boldsymbol{W}}\right|_{\boldsymbol{W}=\boldsymbol{W}^{r}}+\left.\alpha \eta \frac{\partial E_{r}}{\partial \boldsymbol{W}}\right|_{\boldsymbol{W}=\boldsymbol{W}^{r-1}}
\end{gathered}
$$

where $\eta$ is defined as the learning rate, $\alpha$ is the momentum rate and $r$ is the iterative number. Vogl et al [23] proposed a modified method to accelerate the convergence of the BP algorithm. One of the improvements in their algorithm is that the learning rate $\eta$ is varied according to whether or not an iteration decreases the error norm $E_{r}$. If an update results in reduced $E_{r}, \eta$ is multiplied by a factor larger than one for the next iteration. If a step produces a network with the error norm $E_{r}$ more than a few per cent above the previous value, all changes to the weights are rejected, $\eta$ is multiplied by a factor less than one, $\alpha$ is set equal to zero and the step is repeated. This concept was later adopted by Luo and Hanagud [22] in the dynamic learning rate steepest descent (DSD) method they proposed.

The dynamic adjustment of the learning rate is also used in this study. The learning rate, however, is adjusted once every $p$ iterations instead of every iteration. Assuming that the learning rate for the $n$th iteration is represented by $\eta(n)$, this learning rate will be adjusted at the $(n+p)$ th iteration based on the following criterion:

$$
\eta(n+p)=c \eta(n)
$$


where the range of $c$, based on some numerical studies [24], may be selected in the following manner:

$$
c= \begin{cases}1.1-1.3 & \text { if } 0<e_{n}(i)<\varepsilon_{1}, i=1,2, \ldots, p \\ 0.7-0.9 & \text { if (number of negative } \\ & \left.e_{n}(i), i=1,2, \ldots, p\right) \geqslant \varepsilon_{2} \\ 1.0 & \text { if else. }\end{cases}
$$

The error rates $e_{n}(i)$ are defined as

$$
e_{n}(i)=\frac{E_{r}\left(\boldsymbol{W}^{n+i}\right)-E_{r}\left(\boldsymbol{W}^{n+i+1}\right)}{E_{r}\left(\boldsymbol{W}^{n+i}\right)} \quad i=1,2, \ldots, p .
$$

Numerical studies [24] also suggest that $p$ be selected between 10 and $50, \varepsilon_{1}$ between 0.001 and 0.01 and $\varepsilon_{2}$ between $0.1 p$ and $0.5 p$, respectively.

The derivatives in equation (5) are matrices whose arguments can be found as

$$
\frac{\partial E_{r}}{\partial w_{j i}^{k}}=-\delta_{j}^{k} o_{i}^{k-1} .
$$

Note that $o_{i}^{k}$ represents the output from the $i$ th neuron in the $k$ th layer and $\delta_{j}^{k}$ is expressed as

$\delta_{j}^{k}= \begin{cases}\left(y_{j}^{*}-y_{j}\right) f^{\prime}\left(z_{j}^{k}\right) & \text { when } k=3 \\ \left(\sum_{i} \delta_{i}^{k+1} w_{i j}^{k+1}\right) f^{\prime}\left(z_{j}^{k}\right) & \text { when } k \neq 3\end{cases}$

where

$$
z_{j}^{k}=\sum_{i} w_{j i}^{k} o_{i}^{k-1}
$$

and $f^{\prime}\left(z_{j}^{k}\right)$ is the first derivative of the activation function $f(\cdot)$ with respect to ${ }_{j}^{k}$. The activation function used in this study is a sigmoid function.

Riedmiller and Braun [25] indicated that the change of $\Delta w_{i j}$, which is directly related to the training of the $\mathrm{NN}$ model, is not only dependent on the learning rate $\eta$, but also on the partial derivative $\partial E_{r} / \partial w_{j i}^{k}$. It is possible that the effect of the carefully adapted $\eta$ can be drastically disturbed by the unforeseeable behaviour of the derivative itself. In fact, this problem mainly comes from the possible saturation of sigmoid function, i.e. $f^{\prime}\left(z_{j}^{k}\right) \rightarrow 0$ which leads to $\delta_{j}^{k} \rightarrow 0$ and causes the weight matrix to stagnate. To solve this potential problem, Riedmiller and Braun [25] proposed a resilient propagation scheme which directly adapts the weight step based on local gradient information. In this study, we propose the use of a jump factor $\gamma$ in equations (10a) and $(10 b)$ as the following

$\delta_{j}^{k}= \begin{cases}\left(y_{j}^{*}-y_{j}\right)\left[f^{\prime}\left(z_{j}^{k}\right)+\gamma\right] & \text { when } k=3 \\ \left(\sum_{i} \delta_{i}^{k+1} w_{i j}^{k+1}\right)\left[f^{\prime}\left(z_{j}^{k}\right)+\gamma\right] & \text { when } k \neq 3 .\end{cases}$

Numerical studies [24] recommended that the values of $\gamma$ be selected between 0 and 0.15 and can be varied during the training process. The purpose of adding this small positive value to $f^{\prime}\left(z_{j}^{k}\right)$ is to maintain a non-zero value $\delta_{j}^{k}$ and so prevent the weight matrix from stagnating.

\subsection{Selection of training samples}

A well trained $\mathrm{NN}$ model should be able to reflect the characteristics of the system under study. In addition to a robust and efficient training algorithm, the selection of the training samples is another decisive factor in obtaining a representative $\mathrm{NN}$ model. The training samples for the initial off-line training of the $\mathrm{NN}$ model consisted of several sets of input and output data obtained from the FE analyses. In this study, the input data are the modal parameters and the output data are the structural parameters selected to be updated. While the modal parameters are the lowest few natural frequencies and mode shapes, the selection of structural parameters requires some careful consideration. In this study, parametric sensitivity analyses using the FE method are performed in order to identify those parameters that significantly affect the modal parameters of the structure. The sensitivity index $s_{p}^{i}$ between the $i$ th mode natural frequency $f_{i}$ and the structural parameter $g$ is defined as

$$
s_{p}^{i}=\frac{\Delta f_{i} / f_{i}}{\Delta g / g}
$$

where $\Delta f_{i}$ is the variation of $f_{i}$ due to the variation of parameter $\Delta g$.

The structural parameters considered in this study include the material properties, the geometrical properties and the boundary conditions. The boundary conditions usually have a significant influence on the structural dynamic behaviour. The physical modelling of these boundary conditions can range within a spectrum of completely free to completely fixed. Over simplification of the boundary conditions could result in considerable errors between the measured and the calculated results [26]. In this paper, the boundary conditions of a deck at tower and pier supports are modelled by translational and torsional springs with stiffness coefficients of $k_{T}$ and $k_{\theta}$, respectively.

Once the structural parameters are selected, a set of training samples can then be generated. These training samples should cover all possible combinations and ranges of parametric variation to ensure that the $\mathrm{NN}$ model trained using these samples can accurately represent the structural behaviour. In general, if there are $q$ structural parameters to be updated and each parameter has $p$ possible values, then the total number of training samples required to guarantee the sample completeness should be $p^{q}$. For a complex structure, the sample number could be very large and the training becomes computationally intensive if the convergence rate is slow.

Supposing that the ranges within which the structural parameters may vary are known a priori, Atalla and Inmam [20] suggested that a random generation of the structural parameters within the ranges of the variation used to train the network yields the best results. The training data should reflect the probability distribution, such as the Gaussian distribution, of the model parameters being updated. Levin and Lieven [21] proposed a two-part scheme for the selection of training data. The first part consisted of setting each parameter in turn to one of the $p$ possible values while setting all the other parameters to a constant value. The second part consisted of generating 50 training vectors by 


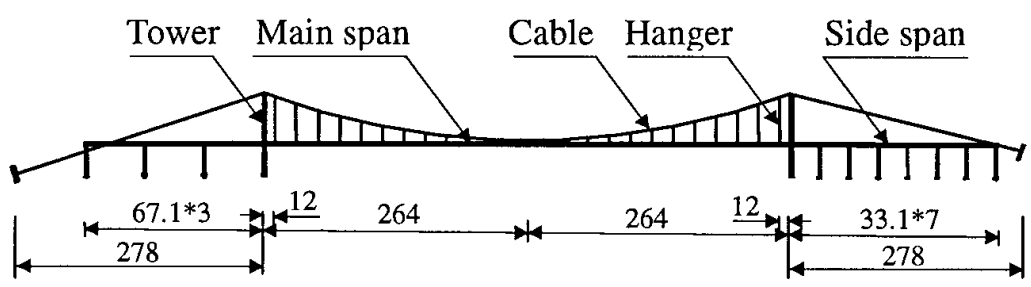

(a)

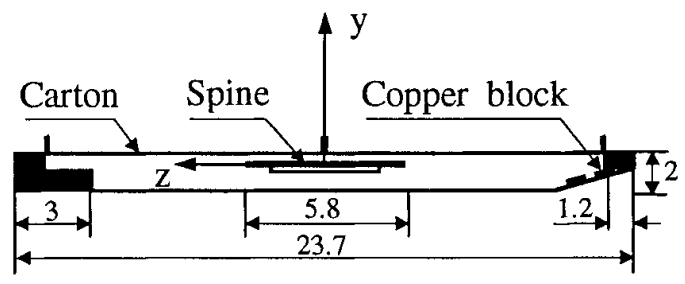

(b)

Figure 4. Humen suspension bridge model (measurements are given in centimetres). (a) Profile of the Humen suspension bridge model, and $(b)$ cross section of the bridge deck.

altering an arbitrary line of parametric values by a random bounded factor. The random samples used in the above two schemes are to represent the sample space spanned by these $q$ parameters, each of which with $p$ possible values (a total of $p^{q}$ samples). This problem actually occurs quite often in quality engineering where experiments involving a few parameters of different values need to be performed in order to find an optimal combination of parameters [27]. The orthogonal array is a method developed for the experimentalist to reduce the number of experimental trials normally required in a full factorial experimental design. The orthogonal array allows an experimentalist to analyse the average change in factor levels under different sets of experimental conditions. In this paper, we adopt this orthogonal array method for the selection of part of the training samples. Assuming that there is no interaction among the $q$ structural parameters selected for updating, the number of samples required based on the orthogonal array [28] for $q$ parameters with $p$ levels is $q(p-1)+1$. This sample number is significantly smaller than the complete sample number of $p^{q}$, especially when the number of structural parameters to be updated $q$ is large. To further reinforce the sample set, it is decided to add another sample group that consists of varying each parameter to their extreme values in turn, while keeping all other parameters at their reference values.

The NN model would need to be re-trained during the updating process if the modal quantities calculated based on the predicted structural parameters from the $\mathrm{NN}$ model do not agree well with the measured ones. Re-training can be achieved by adding new samples to the original pool of samples and enforcing a stringent convergence criterion. It has been pointed out [20] that it could be difficult to achieve the same level of convergence while maintaining the same $\mathrm{NN}$ architecture when the number of samples increases. To this end, a dynamic adjustment method for selecting samples for re-training is proposed. While adding the newly formed sample (based on the structural parameters predicted by the $\mathrm{NN}$ model and the modal parameters output from the FE analyses) into the sample set, one sample from the original sample set is removed so as to keep the same number of samples. The sample removed is the one that has the largest Euclidean distance from the measured modal parameters $\boldsymbol{X}_{m}$. The Euclidean distance of the modal parameters $d$ between the $i$ th sample $\boldsymbol{X}_{i}$ and the measured modal parameters $\boldsymbol{X}_{m}$ is defined as

$$
d=\left\|\boldsymbol{X}_{m}-\boldsymbol{X}_{i}\right\|_{2} .
$$

By replacing this remote sample with a new sample, the sample density around the measured modal parameters would increase as the iteration process progresses. As a result, the modelling accuracy of the NN model in the neighbourhood of the measured modal parameters will improve.

\section{Numerical studies}

Instead of applying the proposed methodology to a real structure in the field, whereby many uncertainties are involved, we consider a structure model tested in the laboratory under a controlled environment. In this connection, a suspension bridge model is built and used to validate the $\mathrm{NN}$ model updating procedure developed above. It is a scaled model of the Humen Bridge located near the city of Taiping in the Pearl Delta region of southern China. The bridge is of a suspension type with a main span of 888 $\mathrm{m}$ and has been in use since 1997 . The laboratory model was built based on the dynamic similitude rules with a geometrical scale of 1:150. The total length of the model is $1025.3 \mathrm{~cm}$ with a centre span of $592 \mathrm{~cm}$ and two side spans of $201.3 \mathrm{~cm}$ and $232.0 \mathrm{~cm}$, respectively (see figure $4(a)$ ). The box girder deck is $23.7 \mathrm{~cm}$ wide and longitudinally separated between the center span and the side spans (see figure 4(b)). The deck is comprised of a spine core, banisters, copper blocks and cartons and is suspended by hangers in the centre span, but supported by piers rather than hangers at both side spans. The height of the towers is $98.4 \mathrm{~cm}$ from the base. The hangers are spaced at $21.3 \mathrm{~cm}$ intervals and the main cable has a sag/span ratio of 1:10.5. The diameter of the steel wire used for cables and hangers is $0.6 \mathrm{~mm}$. Each of the two towers is composed of 15 different rectangular cross 


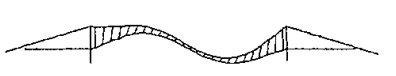

$2.09 \mathrm{~Hz}$

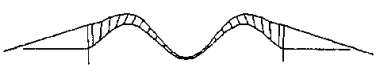

$4.50 \mathrm{~Hz}$

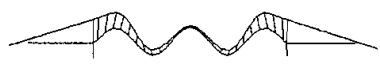

$6.50 \mathrm{~Hz}$

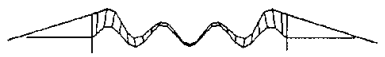

$11.27 \mathrm{~Hz}$

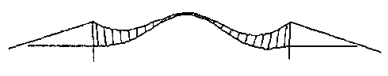

$2.79 \mathrm{~Hz}$

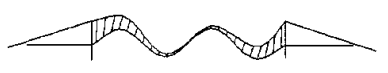

$4.55 \mathrm{~Hz}$

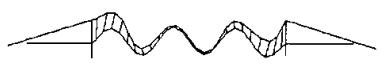

$8.67 \mathrm{~Hz}$

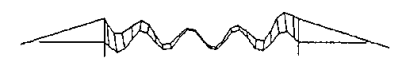

$14.32 \mathrm{~Hz}$
Figure 5. The first eight vertical modes calculated from the FEM.

sections that are made out of steel, lead block, plexiglass and wood. The design material and geometrical properties for the main components of this bridge model are given in table 1 where the modulus of elasticity, the mass density and the cross sectional area are represented by $E, \rho$ and $A$, respectively. Also, the moment of inertias about $y$-axis, about $z$-axis and the polar moment of inertia are represented by $I_{y}$, $I_{z}$ and $J$, respectively. In this study, we only consider the natural frequencies and mode shapes in the vertical direction ( $y$-direction).

An initial (or uncalibrated) three-dimensional FE model was constructed based on the design drawings of the bridge model. Three simplifications are introduced in the FE model: (1) the additional cable tension caused by the inertia forces is negligible, (2) the main cables are assumed to be pinned at the saddle locations of the tower tops and (3) the box-girder deck and the two towers, although made of composites, are represented by an equivalent homogeneous material having the same bending and torsional properties, respectively. All components of the bridge model are represented by beam elements with six degrees of freedom (DOFs) for each node. The geometric stiffness matrices are added to the elastic stiffness for the cables, the hangers and the towers. Each tower is represented by a three-dimensional portal frame with its two legs fixed at the bases. At the locations where the tower legs and the struts are joined, rigid links are added at the ends of the struts to model the encasement of the struts into the tower legs. Rigid links are accordingly assigned to the beam elements to model the joints. The connections between the deck and the hangers are also modelled by rigid elements. The FE model consists of 222 nodes, 208 beam elements, 39 rigid elements, and thus has 1211 DOFs. Considering only the vertical modes, the first eight natural frequencies and the corresponding mode shapes obtained using the FE model are shown in figure 5. These natural frequencies and mode shapes will be used as the inputs to the NN model. Also, after some sensitivity analyses, it was determined that the modal parameters are sensitive to the following structural parameters: elastic modulus $E_{\text {cable }}$ and cross sectional area $A_{\text {cable }}$ for the cable; elastic modulus

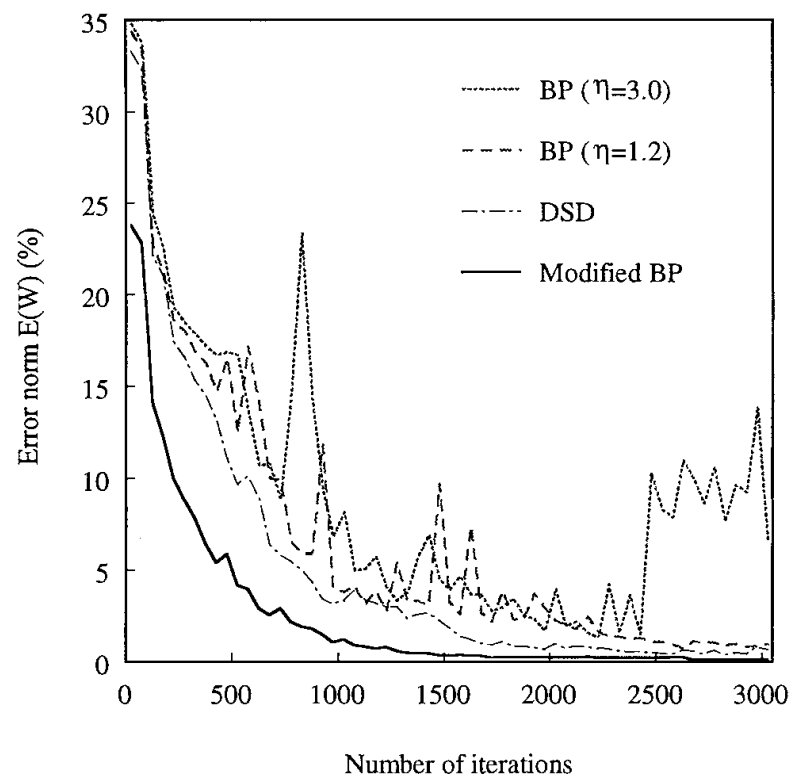

Figure 6. The convergence of the error norm during the initial training of the $\mathrm{NN}$ model.

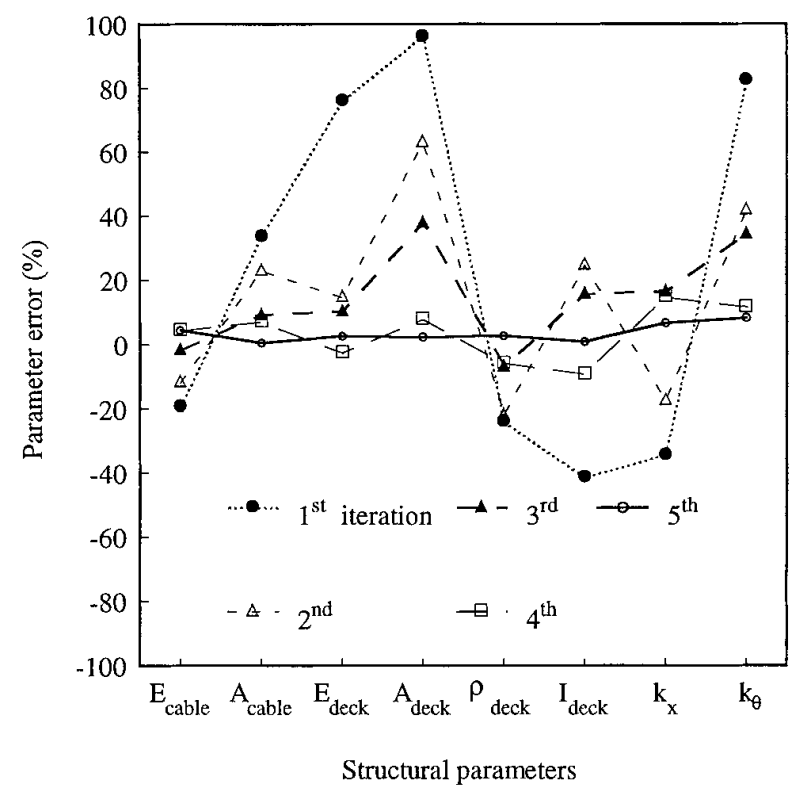

Figure 7. The convergence of the structural parameters for the simulated case.

$E_{\text {deck }}$, cross sectional area $A_{\text {deck }}$, density $\rho_{\text {deck }}$ and bending moment inertia $I_{z \text { deck }}$ for the deck. In addition to these six parameters, it was decided to introduce two spring parameters $k_{x}$ and $k_{\theta}$ to represent the boundary condition of the bridge towers along the longitudinal direction of the deck and the rotational direction at the tower bases, respectively. It is assumed that the boundary conditions at the two tower bases are identical and have the same properties. As a result, there are a total of eight structural parameters selected to be updated in the following studies.

The selected NN model for this study has the same topologic architecture as that as in figure 1, where the node numbers in the input layer, first and second hidden layers and output layer are 16, 39, 16 and 8, respectively. The model is 
Table 1. Properties of the main components for the Humen bridge model.

\begin{tabular}{lllllll}
\hline Component & $\begin{array}{l}E \\
(\mathrm{~Pa})\end{array}$ & $\begin{array}{l}\rho \\
\left(\mathrm{kg} \mathrm{m}^{-3}\right)\end{array}$ & $\begin{array}{l}A \\
\left(\mathrm{~m}^{2}\right)\end{array}$ & $\begin{array}{l}I_{y} \\
\left(\mathrm{~m}^{4}\right)\end{array}$ & $\begin{array}{l}I_{z} \\
\left(\mathrm{~m}^{4}\right)\end{array}$ & $\begin{array}{l}J \\
\left(\mathrm{~m}^{4}\right)\end{array}$ \\
\hline Deck & $1.50 \times 10^{11}$ & $7.50 \times 10^{3}$ & $1.31 \times 10^{-4}$ & $1.75 \times 10^{-10}$ & $1.30 \times 10^{-10}$ & $5.27 \times 10^{-10}$ \\
Cable and suspender & $2.10 \times 10^{11}$ & $7.50 \times 10^{3}$ & $3.06 \times 10^{-7}$ & $1.49 \times 10^{-14}$ & $1.49 \times 10^{-14}$ & $2.98 \times 10^{-14}$ \\
\hline
\end{tabular}

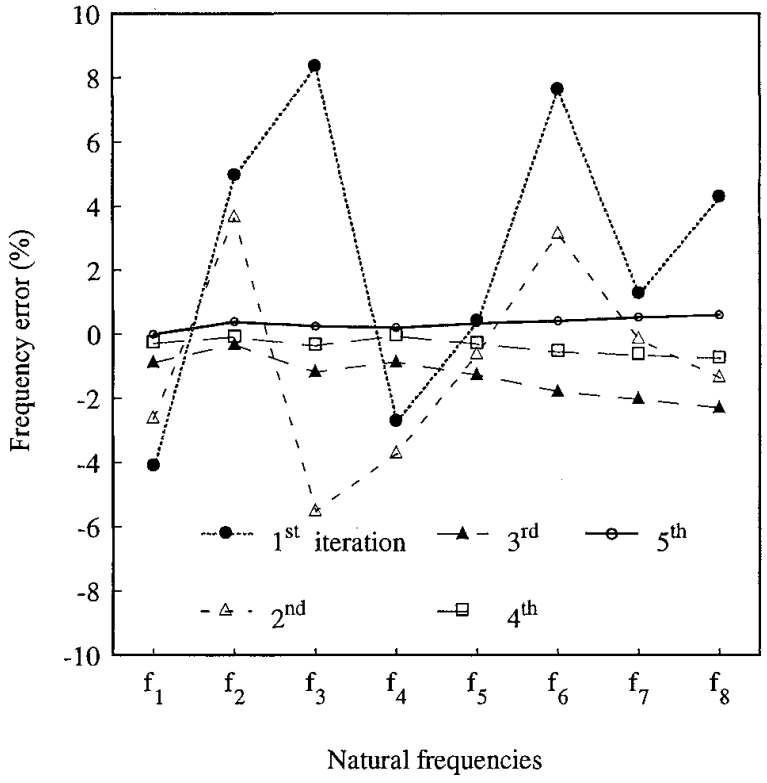

Figure 8. The convergence of the natural frequencies for the simulated case.

trained using the modified BP learning algorithm outlined in the previous section. To formulate the initial training data set, it is assumed that there are three levels of variation for the eight structural parameters: $75 \%, 100 \%$ and $125 \%$ of the design values as outlined in table 1. According to the theory of orthogonal arrays, these eight three-level parameters would require a total of 17 samples to cover the complete sample domain. In addition, another 16 samples, which involve varying each parameter to its extreme values in turn, while keeping all other parameters at their design values, are added to the data set. The corresponding modal parameters of these 33 sets of structural parameters are obtained from the FE analyses using the model outlined above. These training samples are normalized, based on the following formulae before being used in the training process:

$$
\begin{gathered}
\bar{f}_{i}=\frac{f_{i}-0.9 f_{\text {imin }}}{1.1 f_{\text {imax }}-0.9 f_{\text {imin }}} \\
\bar{\phi}_{i}=\frac{\phi_{i}^{T} \phi_{i 0}}{\sqrt{\phi_{i}^{T} \phi_{i}} \sqrt{\phi_{i 0}^{T} \phi_{i 0}}}
\end{gathered}
$$

where $f_{\text {imax }}$ and $f_{\text {imin }}$ are the maximum and minimum values of the $i$ th mode natural frequency respectively in the sample set, $\bar{f}_{i}$ is the normalized $i$ th mode natural frequency of $f_{i}$ ranging between zero and one. The coefficients 0.9 and 1.1 in (15) are to ensure that the normalized values would not be too close to zero or one to avoid numerical difficulties during the training process. The mode shape indexes are also normalized using (16) so as to ensure that they are confined

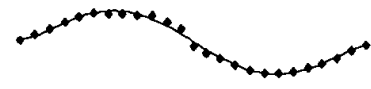

$2.05 \mathrm{~Hz}$

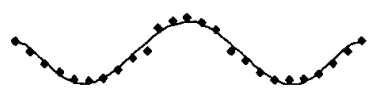

$3.47 \mathrm{~Hz}$

$6.10 \mathrm{~Hz}$

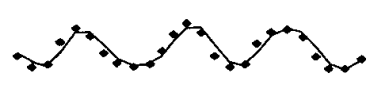

$9.67 \mathrm{~Hz}$

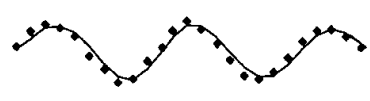

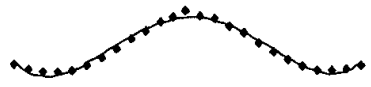

$2.39 \mathrm{~Hz}$
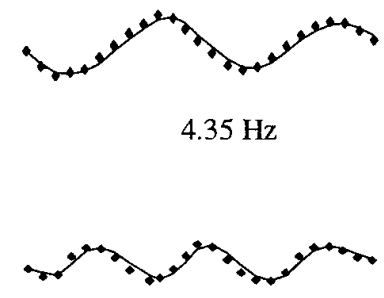

$6.98 \mathrm{~Hz}$

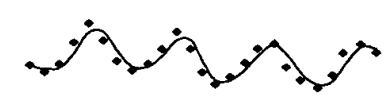

$12.52 \mathrm{~Hz}$
Figure 9. The natural frequencies and mode shapes of the Humen Bridge model obtained from an ambient vibration test.

between zero and one. Note that $\bar{\phi}_{i}$ is the normalized mode shape index for the $i$ th mode shape $\phi_{i}$ and $\phi_{i 0}$ is the $i$ th mode shape obtained from using the set of design parameters in the FE model.

The training of NN model was done in batch, i.e. the weights were only updated after all 33 sets of training data had been presented and processed. Figure 6 shows the convergence of the error norm for the $\mathrm{NN}$ model during the initial training stage. Three training algorithms are used: the original BP method with the learning rate $\eta=3$ and 1.2, respectively; the DSD method with the initial $\eta=2.0$; and the current modified BP algorithm with the initial $\eta=2.5$. The coefficient $c$ used in equations $(7 a)$ and $(7 b)$ is 1.2 and 0.75 , respectively, and the learning rate is adjusted every 30 iterations $(p=30)$. It is seen that, in general, these three training methods all demonstrate a convergent trend as the number of iterations increases. The convergence of the BP method however does not seem to be smooth, especially when $\eta=3$. The dynamic adjustment of the learning rate suggested by the DSD method can actually eliminate the occurrence of ripples as shown when using the BP method. Furthermore, it is seen that the current modified $\mathrm{BP}$ algorithm demonstrates the fastest and the smoothest convergence among the three methods.

\subsection{Simulated case}

To validate the current NN-based model updating procedure, the first eight natural frequencies and mode shapes are first 
Table 2. Model updating for the simulated case.

\begin{tabular}{|c|c|c|c|c|c|c|c|c|}
\hline $\begin{array}{l}\text { Properties or } \\
\text { frequencies }\end{array}$ & $\begin{array}{l}E_{\text {cable }}(\mathrm{Pa}) \\
f_{1}(\mathrm{~Hz})\end{array}$ & $\begin{array}{l}A_{\text {cable }}\left(\mathrm{m}^{2}\right) \\
f_{2}(\mathrm{~Hz})\end{array}$ & $\begin{array}{l}E_{\text {deck }}(\mathrm{Pa}) \\
f_{3}(\mathrm{~Hz})\end{array}$ & $\begin{array}{l}A_{\text {deck }}\left(\mathrm{m}^{2}\right) \\
f_{4}(\mathrm{~Hz})\end{array}$ & $\begin{array}{l}\rho_{\text {deck }}\left(\mathrm{kg} \mathrm{m}^{-3}\right) \\
f_{5}(\mathrm{~Hz})\end{array}$ & $\begin{array}{l}I_{\text {deck }}\left(\mathrm{m}^{4}\right) \\
f_{6}(\mathrm{~Hz})\end{array}$ & $\begin{array}{l}k_{x}\left(\mathrm{~N} \mathrm{~m}^{-1}\right) \\
f_{7}(\mathrm{~Hz})\end{array}$ & $\begin{array}{l}k_{\theta}(\mathrm{N} \mathrm{m}) \\
f_{8}(\mathrm{~Hz})\end{array}$ \\
\hline $\begin{array}{l}\text { Assumed } \\
\text { properties } \\
\text { FEM } \downarrow\end{array}$ & $2.53 \times 10^{11}$ & $3.71 \times 10^{-7}$ & $1.16 \times 10^{11}$ & $1.27 \times 10^{-4}$ & $9.09 \times 10^{3}$ & $1.01 \times 10^{-10}$ & $5.0 \times 10^{8}$ & $5.0 \times 10^{9}$ \\
\hline $\begin{array}{l}\text { Calculated } \\
\text { frequencies } \\
\text { NN } \downarrow\end{array}$ & 1.99 & 2.59 & 4.03 & 4.72 & 5.72 & 7.22 & 9.19 & 11.41 \\
\hline $\begin{array}{l}\text { Properties } \\
\text { (first prediction) } \\
\text { FEM } \downarrow\end{array}$ & $2.04 \times 10^{11}$ & $4.96 \times 10^{-7}$ & $2.04 \times 10^{11}$ & $2.49 \times 10^{-4}$ & $6.91 \times 10^{3}$ & $5.93 \times 10^{-11}$ & $3.28 \times 10^{8}$ & $9.12 \times 10^{9}$ \\
\hline $\begin{array}{l}\text { Calculated } \\
\text { frequencies } \\
\quad \cdots \downarrow\end{array}$ & 1.91 & 2.72 & 4.37 & 4.59 & 5.74 & 7.77 & 9.31 & 11.90 \\
\hline $\begin{array}{l}\text { Properties } \\
\text { (fifth prediction) } \\
\text { FEM } \downarrow\end{array}$ & $2.64 \times 10^{11}$ & $3.73 \times 10^{-7}$ & $1.19 \times 10^{11}$ & $1.30 \times 10^{-4}$ & $9.34 \times 10^{3}$ & $1.02 \times 10^{-10}$ & $5.34 \times 10^{8}$ & $5.42 \times 10^{9}$ \\
\hline $\begin{array}{l}\text { Calculated } \\
\text { frequencies }\end{array}$ & 1.99 & 2.60 & 4.04 & 4.73 & 5.74 & 7.25 & 9.24 & 11.48 \\
\hline
\end{tabular}

Table 3. Model updating for the experimental case.

\begin{tabular}{|c|c|c|c|c|c|c|c|c|}
\hline $\begin{array}{l}\text { Frequencies or } \\
\text { properties }\end{array}$ & $\begin{array}{l}E_{\text {cable }}(\mathrm{Pa}) \\
f_{1}(\mathrm{~Hz})\end{array}$ & $\begin{array}{l}A_{\text {cable }}\left(\mathrm{m}^{2}\right) \\
f_{2}(\mathrm{~Hz})\end{array}$ & $\begin{array}{l}E_{\text {deck }}(\mathrm{Pa}) \\
f_{3}(\mathrm{~Hz})\end{array}$ & $\begin{array}{l}A_{\text {deck }}\left(\mathrm{m}^{2}\right) \\
f_{4}(\mathrm{~Hz})\end{array}$ & $\begin{array}{l}\rho_{\text {deck }}\left(\mathrm{kg} \mathrm{m}^{-2}\right) \\
f_{5}(\mathrm{~Hz})\end{array}$ & $\begin{array}{l}I_{\text {deck }}\left(\mathrm{m}^{4}\right) \\
f_{6}(\mathrm{~Hz})\end{array}$ & $\begin{array}{l}k_{x}\left(\mathrm{~N} \mathrm{~m}^{-1}\right) \\
f_{7}(\mathrm{~Hz})\end{array}$ & $\begin{array}{l}k_{\theta}(\mathrm{N} \mathrm{m}) \\
f_{8}(\mathrm{~Hz})\end{array}$ \\
\hline $\begin{array}{l}\text { Measured } \\
\text { frequencies } \\
\text { NN } \downarrow\end{array}$ & 2.05 & 2.39 & 3.47 & 4.35 & 6.10 & 6.98 & 9.67 & 12.52 \\
\hline $\begin{array}{l}\text { Properties } \\
\text { (first prediction) } \\
\text { FEM } \downarrow\end{array}$ & $1.51 \times 10^{11}$ & $4.38 \times 10^{-1}$ & $1.73 \times 10^{11}$ & $1.91 \times 10^{-4}$ & $6.37 \times 10^{3}$ & $9.76 \times 10^{-11}$ & $4.01 \times 10^{8}$ & $1.25 \times 10^{9}$ \\
\hline $\begin{array}{l}\text { Calculated } \\
\text { frequencies } \\
\quad \cdots \downarrow\end{array}$ & 2.13 & 2.61 & 3.49 & 4.48 & 6.21 & 8.21 & 10.52 & 13.23 \\
\hline $\begin{array}{l}\text { Properties } \\
\text { (fifth prediction) } \\
\text { FEM } \downarrow\end{array}$ & $1.24 \times 10^{11}$ & $3.16 \times 10^{-7}$ & $1.32 \times 10^{11}$ & $1.44 \times 10^{-4}$ & $8.95 \times 10^{3}$ & $1.01 \times 10^{-10}$ & $3.64 \times 10^{8}$ & $8.32 \times 10^{8}$ \\
\hline $\begin{array}{l}\text { Calculated } \\
\text { frequencies }\end{array}$ & 1.97 & 2.49 & 3.40 & 4.18 & 5.71 & 7.43 & 9.42 & 11.71 \\
\hline
\end{tabular}

calculated, based on a set of assumed structural parameters as shown in the first two rows of table 2. The objective of this example is to predict these eight structural parameters using the eight calculated natural frequencies and mode shapes and see whether they match with the assumed values.

The first estimation of the structural parameters is obtained by feeding the 16 normalized modal parameters into the trained NN model. The third row of table 2 indicates the first predicted values. It is seen that the prediction of some parameters, such as $A_{d e c k}$ and $k_{\theta}$, could differ from the assumed values by as much as $100 \%$. It is quite clear that the first prediction is not acceptable. The first predicted structural parameters are then fed into the FE model for the calculation of modal parameters. The results as shown in the fourth row of table 2 indicate that these natural frequencies differ from the reference frequencies in the range of 4-8\%. The retraining process, as outlined in figure 3 , is then conducted. The process is repeated until a set of satisfactory result is obtained. The fifth prediction of the structural parameters shown in the fifth row of table 2 demonstrates that the predicted parameters are already very close to the assumed values. Subsequent calculation of modal parameters shows that the eight natural frequencies match quite well with those calculated based on the assumed properties.
Figures 7 and 8 show the convergence of the structural parameters and the natural frequencies for these five iterations. It is demonstrated that the errors between the predicted and the target values decrease as the number of iteration increases.

\subsection{Experimental case}

To further examine the applicability of the current methodology in the practical situation, the parameters of the scaled-down Humen suspension bridge model are updated and obtained using the actual measurements from the laboratory. An ambient vibration test was performed where an electric fan was used to simulate random loadings on the bridge model. A total of 25 sensor locations distributed along the bridge deck were used to collect the acceleration response of the bridge model. The response was post-processed using an in-house program 'BRIDGEID' to produce natural frequencies, damping ratios and mode shapes. Details of the measurement and the post-processing are outlined in [29].

Figure 9 shows the first eight measured natural frequencies and their corresponding mode shapes for the bridge deck only. These measured frequencies and mode 


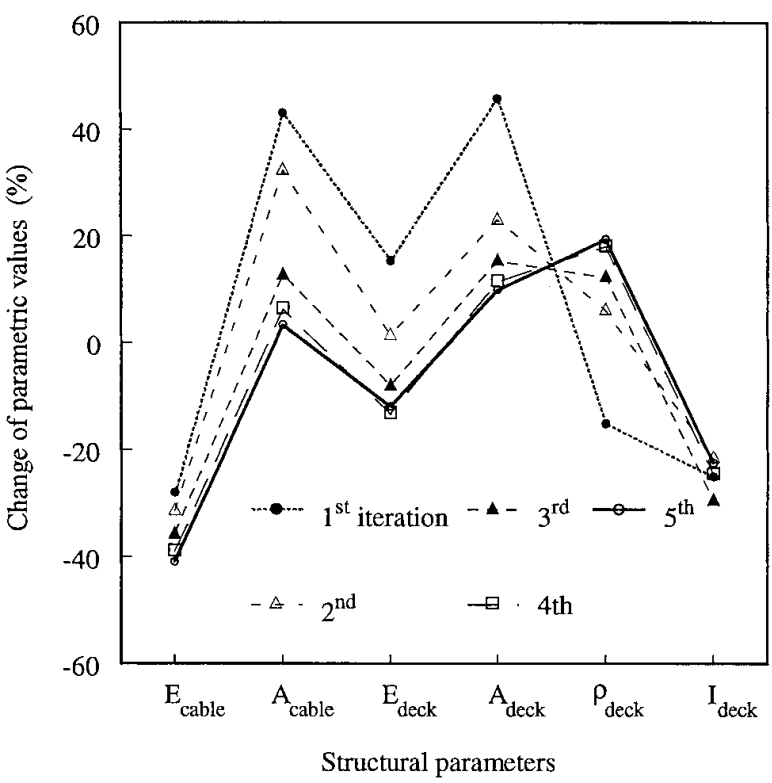

Figure 10. The change of the parametric values for the experimental case.

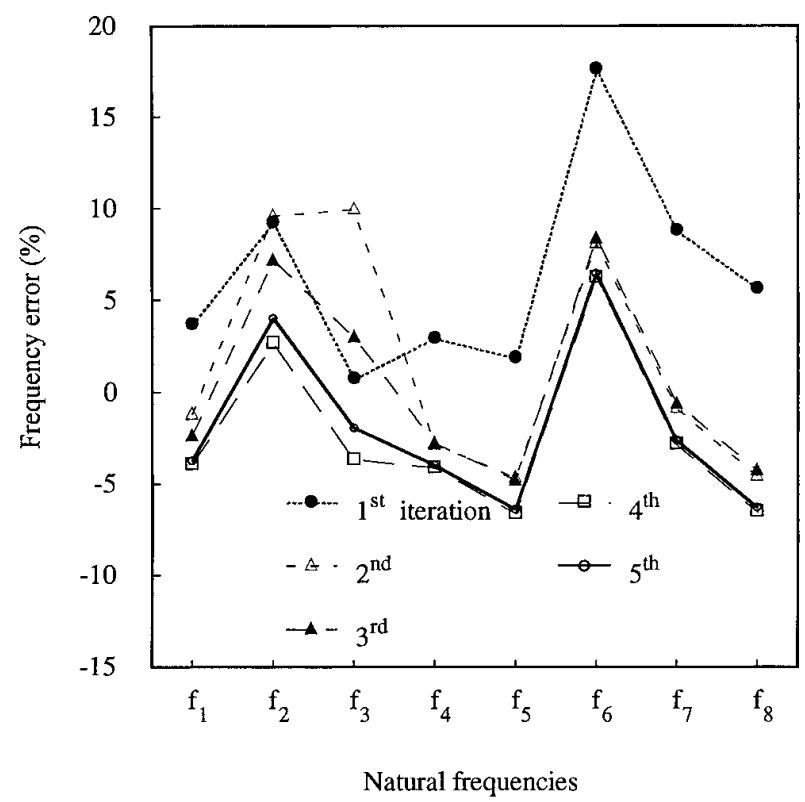

Figure 11. The convergence of the natural frequencies for the experimental case.

shapes are normalized and input into the NN model for the first prediction of the structural parameters. The second row of table 3 shows the first predicted structural parameters. This set of structural parameters is then fed into the FE model to calculate the modal parameters. Again, the retraining process as outlined in figure 3 is necessary. The process continues until no significant changes in the natural frequencies are observed. The final prediction of structural parameters and the natural frequencies calculated based on this set of predictions are also included in table 3. Figure 10 shows the change of parametric values during the updating process. It is found that the updated elastic modulus for the cable $E_{\text {cable }}$ and the updated moment of inertia for the deck $I_{\text {deck }}$ are about $40 \%$ (reduced from $2.10 \times 10^{11}$ to $1.24 \times 10^{11} \mathrm{~Pa}$ ) and $20 \%$ (reduced from $1.30 \times 10^{-10}$ to $1.01 \times 10^{-10} \mathrm{~m}^{4}$ ) less than their design values, respectively. A tension test performed subsequently in the laboratory indicates that the actual elastic modulus of the cable is $1.19 \times 10^{11} \mathrm{~Pa}$. The difference between the updated and the actual values is only about $4 \%$. This is a good indication that the updated result could be quite reasonable.

The convergence of the natural frequencies is shown in figure 11. It is seen that the maximum frequency error between the calculated and the measured frequencies decreases from about $17 \%$ to about $7 \%$. The frequency errors are bounded within $\pm 7 \%$ and further iteration does not seem to reduce the errors. This result can most likely be attributed to the following two reasons.

(1) It has been assumed that the frequency differences come solely from a few selected structural parameters. This assumption excludes some important factors such as the difference between the FE model and the physical model. Although a good and representative FE model should have convergent characteristics as the mesh refines, there is no assurance that they would also converge to that of the physical model even if correct structural parameters are used in the FE model.

(2) Possible errors exist in the measured natural frequencies and mode shapes. These values are obtained from an ambient vibration test. The errors introduced during the measurement and by the post-processing techniques are difficult to quantify.

\section{Concluding remarks}

In this study, a model updating methodology based on an adaptive NN model is proposed. The NN model has a feedforward architecture and is first trained off-line using some training data that are obtained from FE analyses and contain modal parameters as inputs and structural parameters as outputs. To reduce the number of training data while maintaining the data completeness, the variation of structural parameters is arranged using an orthogonal array. This $\mathrm{NN}$ model is then adaptively retrained on-line during the model updating process in order to eliminate the difference between the measured and the predicted modal parameters. A modified BP algorithm is developed, in which the learning rate is dynamically adjusted once every few iterations, and a jump factor is introduced to overcome the numerical difficulty caused by the saturation of the sigmoid function in order to improve the convergence performance of the NN model.

The current adaptive NN updating procedure is applied to a suspension bridge model and verified both numerically and experimentally. The results indicate that by adaptively training the NN model and iteratively adjusting the structural parameters, it is possible to reduce the differences between the measured and the predicted frequencies from a maximum of $17 \%$ to $7 \%$ for the first eight vertical modes.

Based on the current study, some observations on model updating using neural networks can be drawn as the following. 
(1) The NN approach is a viable tool for model updating of complex structures. It has the advantage of updating directly the structural parameters, including physical and geometrical parameters and boundary conditions, based on measured information.

(2) Training of the NN model usually is very timeconsuming especially when the number of training samples is large. The orthogonal arrays that are frequently used in quality engineering were adopted and found to be useful in drastically reducing the number of samples without sacrificing sample completeness.

(3) By allowing the learning rate to be dynamically adjusted and by introducing a jump factor the adaptive learning algorithm can significantly improve the convergence performance of the network training.

(4) The simulated results demonstrate that the current adaptive NN approach can be quite accurate. When used in the laboratory study, the approach can reduce the frequency differences between the measured and the predicted values, although complete matching does not seem to be likely. The reason could be due to the over simplification of a far more complex problem in which the frequency differences are assumed to be contributed solely from the miss-match of a few structural parameters.

\section{Acknowledgments}

Research work reported in this paper is supported by a competitive Earmarked Grant for Research, the Research Grants Council of Hong Kong and a collaborated research grant UIC99/98.EG01 funded by the NSF via the University of Illinois at Chicago.

\section{References}

[1] Friswell M I and Mottershead J E 1995 Finite element model updating in structural dynamics (Dordrecht: Kluwer)

[2] Baruch M 1982 Optimal correction of mass and stiffness matrices using measured modes AIAA J. 20 1623-6

[3] Caesar B 1987 Updating system matrices using modal test data Proc. 5th Int. Modal Analysis Conf. (London, UK, April 6-9, 1987) pp 453-9

[4] Minas C and Inman D J 1988 Correcting finite element models with measured modal results using eigenstructure assignment methods Proc. 6th Int. Modal Analysis Conf. (Orlando, USA) pp 583-7

[5] Starek L and Inman D J 1991 Solution of the model correction problem via inverse methods Proc. 9th Int. Modal Analysis Conf. (Florence, Italy, April 15-18, 1991) pp 352-5

[6] Link M 1993 updating of analytical models-procedures and experience Proc. Conf. on Modern Practice in Stress and Vibration Analysis (Sheffield, UK) pp 35-52

[7] Eckert L and Caesar B 1991 Model updating under incomplete and noisy modal test data Proc. 9th Int. Modal Analysis Conf. (Florence, Italy, April 15-18, 1991) pp 563-71

[8] Collins J D, Hart G C, Hasselman T K and Kennedy B 1974 Statistical identification of structures AIAA J. 12 185-90
[9] Hemez F M and Farhat C 1993 Locating and identifying structural damage using a sensitivity-based model updating methodology 34th AIAA/ASME/ASCE/AMS/ASC Structures, Structural Dynamic and Materials Conf. (La Jolla, DC, USA, April 19-22, 1993) pt 5, pp 2641-53

[10] Tan R C E 1989 Some acceleration methods for the iterative computation of derivatives of eigenvalues and eigenvectors Int. J. Num. Methods Engng 28 1505-19

[11] Mottershead J E, Friswell M I and Ahmadian H 1998 Cross validation and $\mathrm{L}$-curves for the regularization of ill-conditioned equations in model updating Proc. 16th Int. Modal Analysis Conf. (Bethel, CT, USA, February 2-5, 1998) pp 1333-7

[12] Ahmadian H, Mottershead J E and Friswell M I 1998 Regularization methods for finite element model updating Mech. Syst. Signal Process. 12 47-64

[13] Fritzen C P, Jennewein D and Kiefer T 1998 Damage detection based on model updating methods Mech. Syst. Signal Process. 12 163-86

[14] Adeli H and Yeh C 1989 Perceptron learning in engineering design Microcomput. Civil Engng 4 247-56

[15] Wu X, Ghaboussi J and Garrett J H 1992 Use of neural networks in detection of structural damage Comput. Struct. 42 649-59

[16] Hajela P and Berke L 1991 Neurobiological computational modes in structural analysis and design Comput. Struct. $41657-67$

[17] Masri S F, Chassiakos A G and Caughey T K 1993 Identification of nonlinear dynamics system using neural networks J. Appl. Mech. 60 123-33

[18] Stephens J E and Vanluchene R D 1994 Integrated assessment of seismic damage in structures Microcomput Civil Engng 9 119-28

[19] Elkordy M F, Chang K C and Lee G C 1994 A structural damage neural network monitoring system Microcomput. Civil Engng 9 83-96

[20] Atalla M J and Inman D J 1998 On model updating using neural networksMech. Syst. Signal Process. 12 135-61

[21] Levin R I and Lieven N A J 1998 Dynamic finite element model updating using neural networks J. Sound Vib. 210 593-607

[22] Luo H and Hanagud S 1997 Dynamic learning rate neural networks training and composite structural damage detection AIAA J. 35 1522-7

[23] Vogl T P, Mangis J K, Rigler A K, Zink W T and Alkon D L 1988 Accelerating the convergence of the back-propagation method Biol. Cybernet. 59 257-63

[24] Xu Y 1996 Research on the dynamic intelligent diagnosis and reliability evaluation for structures $P h D$ Thesis Huazhong University of Science and Technology, Wuhan, China

[25] Riedmiller M and Braun H 1993 A direct adaptive method for faster backpropagation learning: the RPROP algorithm Proc. IEEE Int. Conf. on Neural Networks (Nagoya, Japan, October 25-29, 1993) pp 586-91

[26] Ahmadian H, Gladwell G M L and Ismail F 1997 Parameter selection strategies in finite element model updating $J$. Vib. Acoust.-Trans. ASME 119 37-45

[27] Ealey L A 1994 Quality by Design: Taguchi Methods and US Industry 2nd edn (Burr Ridge, IL: Irwin Professional)

[28] Besterfield D H, Besterfield-Michna C, Besterfield G H and Besterfield-Sacre M 1995 Total Quality Management (Englewood Cliffs, NJ: Prentice-Hall)

[29] Chang C C, Chang T Y P and Zhu L D 199 A streamlined ambient vibration analysis procedure for long-span bridges Proc. 3rd Int. Symp. on Civil Infrastructure Systems: Intelligent Renewal (Capri, Italy, September 24-26, 1997) pp 57-71 